DOI: $10.1002 /$ marc.201400714

\title{
Communication
}

\section{Triplet state formation in photovoltaic blends of DPP-type copolymers and $\mathbf{P C}_{71} \mathbf{B M}^{\mathrm{a}}$}

Julian R. Ochsmann, Deepak Chandran, Dominik W. Gehrig, Husna Anwar, Pramod Kandoth Madathil, Kwang-Sup Lee, ${ }^{*}$ and Frédéric Laquai*

J. R. Ochsmann, D. W. Gehrig, Dr. F. Laquai

Max Planck Research Group for Organic Optoelectronics, Max Planck Institute for Polymer Research, Ackermannweg 10, 55128 Mainz, Germany

E-mail: laquai@mpip-mainz.mpg.de

Assoc. Prof. F. Laquai

present address: Physical Sciences and Engineering Division (PSE), Material Science and Engineering (MSE), Solar and Photovoltaics Engineering Research Center (SPERC), King Abdullah University of Science and Technology (KAUST), Thuwal 23955-6900, Kingdom of Saudi Arabia

E-mail: frederic.laquai@kaust.edu.sa

H. Anwar

Department of Physics, Mount Holyoke College, 50 College St, South Hadley, MA 01075, USA

Dr. D. Chandran, Dr. P. K. Madathil, Prof. Dr. K.-S. Lee

Department of Advanced Materials, Hannam University, Daejeon 305-811, Korea E-mail:kslee@mail.hannam.ac.kr

Dr. D. Chandran

present address: School of Chemical Sciences \& National Institute for Cellular Biotechnology, Dublin City University, Glasnevin, Dublin-9, Ireland

The exciton dynamics in pristine films of two structurally-related low-bandgap DPP-based donor-acceptor copolymers and the photophysical processes in bulk heterojunction solar cells using DPP-copolymer: $\mathrm{PC}_{71} \mathrm{BM}$ blends are investigated by broadband transient absorption pump-probe experiments covering the Vis-NIR spectral and fs- $\mu$ s dynamic range. The

\footnotetext{
${ }^{\text {a }}$ Supporting Information is available online from the Wiley Online Library or from the author.
} 
experiments reveal surprisingly short exciton lifetimes in the pristine polymer films in conjunction with fast triplet state formation. An in-depth analysis of the TA data by multivariate curve resolution (MCR) analysis shows that in blends with fullerene as acceptor ultrafast exciton dissociation creates charge carriers, which then rapidly recombine on the sub-ns timescale. Furthermore, at the carrier densities created by pulsed laser excitation the charge carrier recombination leads to a substantial population of the polymer triplet state. In fact, virtually quantitative formation of triplet states is observed on the ns timescale. However, the quantitative triplet formation on the sub-ns timescale is not in line with the power conversion efficiencies of devices indicating that triplet state formation is an intensitydependent process in these blends and is reduced under solar illumination conditions, as free charge carriers can be extracted from the photoactive layer in devices. 


\section{Introduction}

Substantial research efforts have been devoted to the development of a better understanding of the physics of organic solar cells and to improving their efficiency, as they offer the potential of energy efficient and low-cost large-area production, are intrinsically light-weight and allow the production of flexible devices. A promising approach to increase the efficiency of organic solar cells is to use low band-gap materials as absorbers, since they increase the overlap of the photoactive layer's absorption with the solar spectrum in the low-energy NIR wavelength region and thereby increase the photon collection of the device. Recently, low band-gap materials based on electron-deficient diketopyrrolopyrrole (DPP) building blocks as acceptors have been very successfully introduced for application in solution-processed organic bulk heterojunction $(\mathrm{BHJ})$ solar cells and organic thin film transistors (OTFT). ${ }^{[1,2]}$ Their high charge carrier mobility, strong absorption and stability make them excellent candidates for both, OPV and OTFT devices. ${ }^{[3-13]}$ In single layer bulk heterojunction OPV devices several

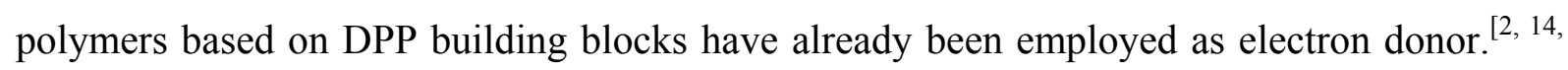
${ }^{15]}$ Furthermore, they have been successfully used in tandem and triple junction solar cells, as they have the advantage of absorbing light mostly in the NIR spectral region, complementary to the absorption of some ubiquitous mid-bandgap polymers such as P3HT or PCDTBT and still yield high photocurrents which allows for current matching in multijunction devices. In fact, tandem devices in which one cell is based on the DPP-type donor polymer PDPP5T in combination with PCDTBT:PCBM or P3HT:PCBM subcells have shown power conversion efficiencies of $7.0 \%$ and $7.23 \%$, respectively. ${ }^{[16,17]}$ In this study, we report the photovoltaic performance of two DPP-based copolymers and we study their excited state dynamics, namely the exciton and charge carrier dynamics, in pristine films and in blends with $\mathrm{PC}_{71} \mathrm{BM}$ as electron acceptor as typically used in the photoactive layer of single junction organic BHJ solar cells. The first polymer under investigation is poly[3,6-dithiene-2-yl-2,5-di(2decyltetradecyl)-pyrrolo[3,4-c]pyrrole-1,4-dione-5',5"-diyl-alt-thieno[3,2-b]thienyl] 
(PTDPP-TT), which consists of alternating DPP building blocks and 2,5-di-2thienylthieno[3,2-b]thiophene units along the polymer backbone. The second polymer studied herein is PFDPP-TT, which has a similar chemical structure as PTDPP-TT, except that the thiophenes adjacent to the DPP-moiety were replaced by furan (see scheme 1).

a)

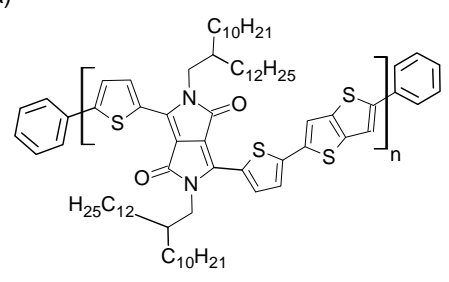

b)

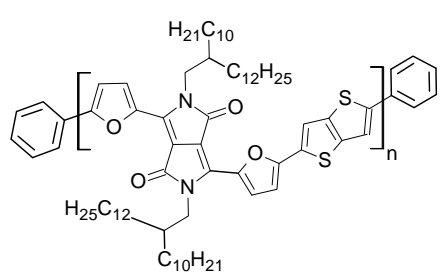

Scheme 1. Chemical structure of a) PTDPP-TT and b) PFDPP-TT.

Janssen and coworkers very recently studied a related polymer, namely DT-PDPP2T-TT, which in fact has the same chemical structure as PTDPP-TT presented in this study, but in their case had a significantly higher molecular weight of $M_{n}=447 \mathrm{~kg} \mathrm{~mol}^{-1}$ compared to $\mathrm{M}_{\mathrm{n}}=47 \mathrm{~kg} \mathrm{~mol}^{-1}$ for our PTDPP-TT polymer as determined by high-temperature GPC. ${ }^{[18]}$ They demonstrated that in a BHJ solar cell architecture the DT-PDPP2T-TT polymer blended with $\mathrm{PC}_{71} \mathrm{BM}$ as acceptor exhibits high power conversion efficiencies even for relatively large $(\sim 300 \mathrm{~nm})$ active layer thicknesses, which makes this polymer particularly interesting for large-area production processes such as inkjet printing and roll-to-roll processing. However, here we focus on the excited state dynamics occurring after pulsed laser excitation of the pristine polymer films and photovoltaic blends with PCBM studied by Vis-NIR broadband pump-probe transient absorption (TA) spectroscopy covering the ps- $\mu$ s dynamic range, that is the dynamic range relevant for exciton recombination, exciton dissociation, charge carrier separation leading to free charge generation, as well as geminate and non-geminate recombination processes. Transient absorption (TA) spectroscopy has previously been applied to gain a better understanding of the fundamental processes determining the efficiency of DPP-polymer:fullerene photoactive blends. For instance, Durrant and coworkers have shown 
in different studies that excess energy is beneficial for charge separation in certain DPP-type polymers $^{[19]}$ and that in some blends photons absorbed by the DPP-polymer do not generate charges due to a lack of an energy offset between donor and acceptor. ${ }^{[20]}$ Here, we specifically address in more detail triplet state formation in DPP-polymer blends observed under pulsed laser excitation. Triplet state formation in photovoltaic blends has recently gained attention in some other low-bandgap polymer:fullerene systems and different mechanisms such as recombination of bound charge-transfer (CT) states and non-geminate recombination of free charges leading to the population of the triplet manifold are currently discussed. ${ }^{[21-23]}$ Triplet exciton generation was previously discussed for DPP-type polymers, but TA experiments in nitrogen and oxygen atmosphere showed identical signal decay dynamics and thus triplet formation was excluded. However, the triplet energy of the polymer has to be $0.98 \mathrm{eV}$ or above for triplet states to be quenched by oxygen, which may not be the case for the DPPpolymer triplet state. ${ }^{[24]}$ In fact, here we show that triplet states are also generated in DPP-type polymer:fullerene blends and become the dominant species present on the ns- $\mu$ s timescale after pulsed laser excitation. We discuss the mechanism of triplet state formation and its impact on device performance under solar illumination conditions. 


\section{Results and Discussion}

\subsection{Photovoltaic Performance}

a)

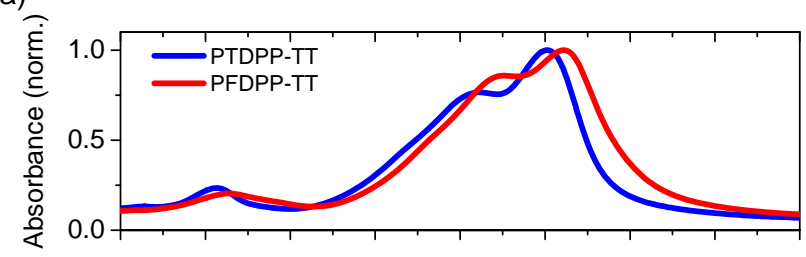

b)

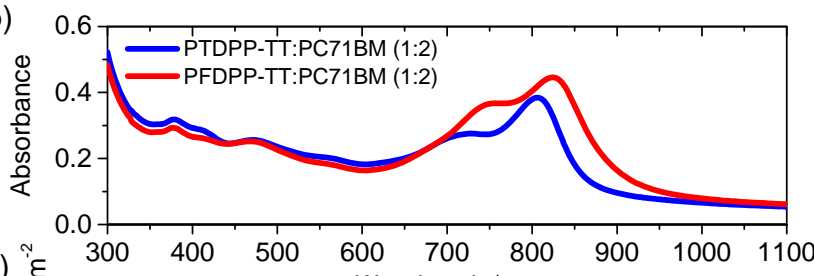

c)

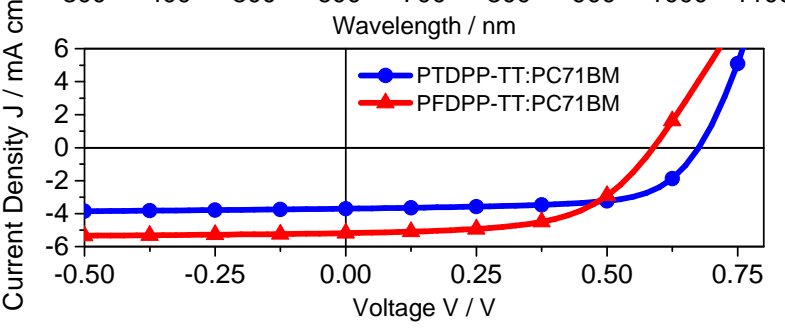

Figure 1. a) Steady-state UV-vis absorption spectra of a pristine PTDPP-TT film (blue line) and PFDPP-TT film (red line). b) Absorption spectrum of a 1:2 (w/w) PTDPP-TT:PC ${ }_{71} B M$ (blue curve; $\mathrm{d} \sim 83 \mathrm{~nm}$ ) and a 1:2 (w/w) PFDPP-TT:PC ${ }_{71} \mathrm{BM}$ blend film (red curve; $\mathrm{d} \sim 87 \mathrm{~nm}$ ) c) J-V characteristics of solar cells using a 1:2 (w/w) PTDPP-TT:PC ${ }_{71} B M$ (blue curve) blend and a 1:2 (w/w) PFDPP-TT:PC ${ }_{71} \mathrm{BM}$ (red curve) blend measured at an illumination of $73 \mathrm{~mW}$ $\mathrm{cm}^{-2}$.

Figure 1a shows the absorption spectra of pristine PTDPP-TT (blue) and pristine PFDPP-TT (red) thin films. Both polymers show a broad and weakly-structured absorption covering the wavelength region from 600 to $900 \mathrm{~nm}$ and a considerably weaker absorption band at around $400 \mathrm{~nm}$. The absorption maximum of PFDPP-TT is at $825 \mathrm{~nm}$, which is slightly red-shifted compared to the absorption maximum of PTDPP-TT around $800 \mathrm{~nm}$. The red-shifted absorption onset and concomitantly smaller band-gap of PFDPP-TT in comparison to PTDPP-TT is likely due to the difference in molecular structure, that is, the substitution of thiophene with furan, which increases the electron density in the polymer backbone due to the electron-donating effect of the lone pairs of the furan oxygen atoms. The absorption spectra of 
polymer: $\mathrm{PC}_{71} \mathrm{BM}$ blend films are presented in figure $1 \mathrm{~b}$. The spectra exhibit similar features as the pristine polymer films, with the additional absorption of $\mathrm{PC}_{71} \mathrm{BM}$ superimposed on the polymer absorption in turn causing a higher absorbance at shorter wavelengths, which extends into the UV spectral region. Hence, both polymer:fullerene blends cover a considerable part of the solar spectrum from the UV- to the NIR wavelength region, which is beneficial for solar energy conversion.

The current-voltage (J-V) characteristics of as-cast bulk heterojunction (BHJ) solar cells built with PTDPP-TT:PC ${ }_{71} \mathrm{BM}$ (1:2 by weight, blue curve) and PFDPP-TT:PC ${ }_{71} \mathrm{BM}$ (1:2 by weight, red curve) using a solar illumination of $73 \mathrm{~mW} \mathrm{~cm}^{-2}$ are depicted in figure $1 \mathrm{c}$. The optimal photovoltaic performance for both material systems was achieved by spincoating the blends from chloroform after an addition of $2 \%$ dioodooctane (DIO) per volume as solvent additive. In case of the PTDPP-TT:PC ${ }_{71} \mathrm{BM}$ system an average power conversion efficiency (PCE) $\eta$ of $1.9 \pm 0.3 \%$ was achieved with a $75 \mathrm{~nm}$ thick photoactive layer. The corresponding open circuit voltage $\left(V_{O C}\right)$ was $0.67 \pm 0.01 \mathrm{~V}$, the short circuit current $\left(J_{S C}\right)$ was $3.4 \pm 0.5 \mathrm{~mA} \mathrm{~cm}^{-2}$ and the fill factor $(F F)$ was $0.62 \pm 0.04$. The best photovoltaic performance for the PFDPP-TT:PC 71 BM system was obtained for an active layer thickness of $82 \mathrm{~nm}$, yielding an average PCE of $2.2 \pm 0.2 \%$, however, a lower $V_{O C}$ of $0.59 \pm 0.00 \mathrm{~V}$, but a higher $J_{S C}$ of $4.7 \pm 0.4 \mathrm{~mA} \mathrm{~cm}^{-2}$ and a slightly lower $F F$ of $0.58 \pm 0.02$ in comparison to the PTDPP-TT:PC ${ }_{71} \mathrm{BM}$ material system. The difference in average $J_{S C}$ is well in line with a reduced EQE of PTDPP-TT:PC ${ }_{71} \mathrm{BM}$ devices across the entire absorption of the photoactive layer. The theoretical $\mathrm{J}_{\mathrm{SC}}$ calculated from the absorption profiles of PTDPP-TT:PC ${ }_{71} \mathrm{BM}$ and PFDPP-TT:PC ${ }_{71} \mathrm{BM}$ assuming $100 \%$ IQE is $14 \mathrm{~mA} \mathrm{~cm}^{-2}$ and $15.8 \mathrm{~mA} \mathrm{~cm}^{-2}$, respectively, indicating substantial loss processes occur in these devices. We also note, that the photovoltaic performance of both systems could neither be further improved by annealing of the photoactive layer nor by using a $\mathrm{Ca} / \mathrm{Al}$ electrode instead of an aluminum electrode only, 
indicating that electron extraction from the photoactive layer is not a significant efficiencylimiting issue in these blends.

\subsection{Exciton Dynamics in Pristine PTDPP-TT and PFDPP-TT Films}

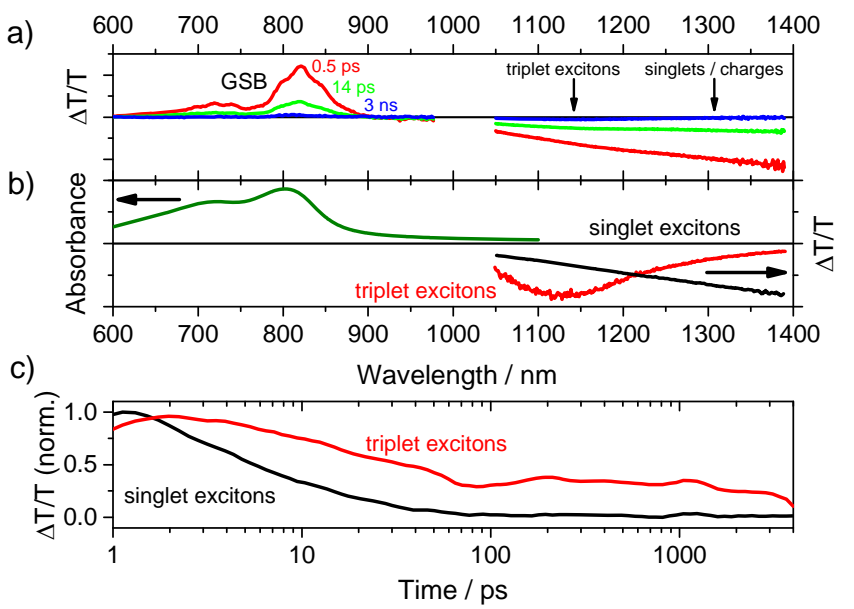

Figure 2. a) ps-ns Vis-NIR transient absorption spectra of a pristine PTDPP-TT film at delay times of $0.5 \mathrm{ps}$ (red), $14 \mathrm{ps}$ (green) and $3 \mathrm{~ns}$ (blue). Excitation was performed at $800 \mathrm{~nm}$ with $5.7 \mu \mathrm{J} \mathrm{cm}^{-2}$ for the visible and $6 \mu \mathrm{J} \mathrm{cm}^{-2}$ for the NIR.

b) Absorbance of the polymer film (dark green), singlet-induced absorption spectrum obtained from MCR analysis (black), and triplet-induced absorption spectrum (red) obtained on a palladium-anthraporphyrin-doped polymer film $(95: 5 \mathrm{w} / \mathrm{w})$.

c) Dynamics of singlet (black) and triplet (red) excitons obtained by MCR analysis using the separately determined triplet-induced absorption spectrum as input parameter (see figure $2 b$ ).

Figure 2a shows the short delay (ps-ns) transient absorption pump-probe spectra of a PTDPP-

TT film in both the visible $(600-1000 \mathrm{~nm})$ and NIR $(1150-1400 \mathrm{~nm})$ probe region. The spectra consist of a positive feature roughly in the spectral region from 650 to $900 \mathrm{~nm}$, which we assign to the polymer's ground state bleach (GSB), since it displays the same spectral position and features as the steady-state absorption of the polymer film also shown in figure $2 \mathrm{~b}$ for comparison (dark green curve). The TA spectrum in the NIR wavelength range exhibits a broad photoinduced absorption (PA) signal, which extends from $1050 \mathrm{~nm}$ further into the NIR. These spectral features are most pronounced shortly after excitation (red curve). 
However, after only 14 ps most of the signals have already substantially decayed (green curve) and after $3 \mathrm{~ns}$, that is the longest delay time used in our ps-ns experiment, the TA signals have almost vanished. All signals in the ps-ns time regime, namely the GSB and PA, are predominantly caused by the presence of singlet excitons in the pristine polymer films, which exhibit a surprisingly short life time in this polymer compared to other common conjugated polymers. ${ }^{[25,26]}$ However, we note that after 3 ns roughly $5 \%$ of the PA signal intensity remain. This residual signal, which peaks at $1150 \mathrm{~nm}$ in the pristine polymer film, is assigned to triplet excitons, as its spectral signature is very similar to the photoinduced absorption of a palladium-anthraporphyrin-doped polymer film (see figure 2c). Here the palladium-anthraporphyrin effectively sensitizes the polymer's triplet level by successive singlet energy transfer to, and intersystem crossing on the sensitizer followed by triplet energy transfer to the polymer. Surprisingly, in the pristine polymer films triplets are created on a very short timescale, which could indicate ultrafast singlet fission. To further analyze the photophysical processes in the pristine polymer film and to obtain the individual dynamics of singlet and triplet states, we performed multivariate curve resolution (MCR) analysis on the TA data, a soft modeling technique, whose application to the analysis of TA data has recently been presented and discussed by us in greater detail. ${ }^{[27]}$ At first we performed MCR analysis without any further constraints (such as spectra and non-negativity of concentrations) and in a second run confined the triplet-induced absorption spectrum to the independently-measured triplet-induced absorption spectrum (see figure $2 \mathrm{c}$ ). While the spectra obtained by MCR analysis show some differences depending on the constraints, the dynamics of the singlet decay and triplet formation are virtually the same (see SI), which supports our data analysis and interpretation. The singlet decay can be fit by two exponentials having inverse decay rates of 3 ps and $17 \mathrm{ps,} \mathrm{the} \mathrm{former} \mathrm{actually} \mathrm{very} \mathrm{well} \mathrm{in} \mathrm{line} \mathrm{with} \mathrm{the} \mathrm{rise} \mathrm{of} \mathrm{the} \mathrm{triplet-induced}$ absorption. This ultrafast triplet formation likely indicates singlet fission in these materials as it appears to be too fast for intersystem crossing. The reason for the rather short singlet 
exciton lifetime of $\sim 17 \mathrm{ps}$ in the pristine polymer films requires further studies, here we can only speculate that it is due to fast and radiationless internal conversion of excited states to the ground state, possibly via a conical intersection between the two states. We note that the decay of singlet states is virtually similar at higher fluences indicating that the dynamics are not influenced by any higher order processes such as exciton-exciton annihilation. We have also analyzed the dynamics of the triplet-induced absorption in a metallated porphyrinsensitized sample and obtained a surprisingly short triplet state lifetime of only about 15 ns by fitting the dynamics to a single exponential decay (see Figure S3). This indicates fast quenching of the triplet states in the polymer, as the triplet lifetime is unusually short compared to other polymers that we have previously investigated. Virtually similar observations regarding the singlet state lifetime and triplet state formation were made for the structurally-related PFDPP-TT polymer. The corresponding spectra for this polymer are also shown in the SI as figure S2. The inverse decay rates obtained for the singlet exciton decay in pristine PFDPP-TT are 3 ps and 19 ps, respectively. Here, the triplet state lifetime obtained from the sensitizer-doped polymer film was determined to be $\sim 11$ ns (see Figure S4). In conclusion, firstly both polymers exhibit a very similar, but rather short singlet exciton lifetime, which is likely caused by fast non-radiative deactivation (or internal conversion) of excited singlet states and secondly a small fraction of triplets is created on a very short timescale pointing to fission of singlet states in these materials. In addition to the very short singlet exciton lifetimes of less than 20 ps, we also found surprisingly short triplet states lifetimes in palladium anthraporphyrin-doped polymer films of $15 \mathrm{~ns}$ and $11 \mathrm{~ns}$ for PTDPPTT and PFDPP-TT, respectively. 


\subsection{Photophysics of PTDPP-TT:PC ${ }_{71} \mathrm{BM}$ and PFDPP-TT:PC ${ }_{71} \mathrm{BM}$ Blends}

a)

b)

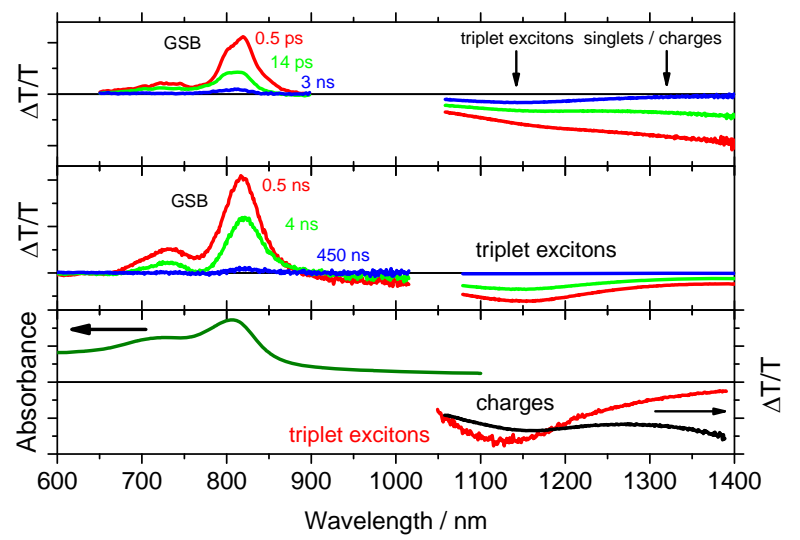

d)

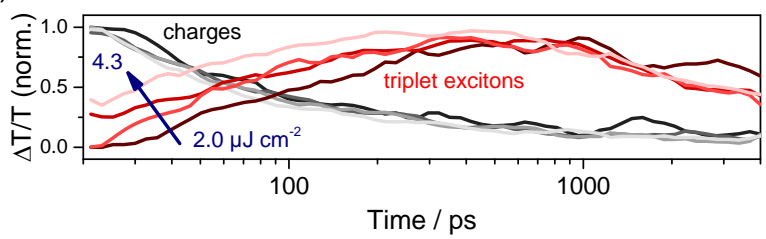

Figure 3. a) ps-ns Vis-NIR transient absorption spectra of a 1:2 (w/w) PTDPP-TT:PC ${ }_{71} B M$ blend at delay times of $0.5 \mathrm{ps}$ (red), $14 \mathrm{ps}$ (green) and $3 \mathrm{~ns}$ (blue). The pump fluence at $800 \mathrm{~nm}$ was $2.7 \mu \mathrm{J} \mathrm{cm}^{-2}$ for the visible and $2 \mu \mathrm{J} \mathrm{cm}^{-2}$ for the NIR measurements. b) ns- $\mu$ s Vis-NIR transient absorption spectra of a 1:2 (w/w) PTDPP-TT:PC ${ }_{71}$ BM blend at delay times of $0.5 \mathrm{~ns}$ (red), $4 \mathrm{~ns}$ (green) and $450 \mathrm{~ns}$ (blue). The pump fluence at $532 \mathrm{~nm}$ was $74.8 \mu \mathrm{J} \mathrm{cm}^{-2}$ for the visible and $36.7 \mu \mathrm{J} \mathrm{cm}^{-2}$ for the NIR measurements. c) Absorption spectrum of a PTDPP-TT:PC ${ }_{71} \mathrm{BM}$ blend film (dark green), charge-induced absorption spectrum as obtained from MCR analysis (black), and triplet-induced absorption spectrum (red) obtained on a palladium-anthraporphyrin-doped polymer film. d) Fluence dependence of the dynamics of charges (black) and triplet excitons (red) obtained by MCR analysis using the separately determined triplet-induced absorption spectrum as input parameter (compare panel c, red curve). The corresponding charge spectrum is shown in panel c (black curve). Only data after 20 ps was used to exclude contributions from singlet excitons.

Figure $3 \mathrm{a}$ and $3 \mathrm{~b}$ display the Vis-NIR pump-probe spectra of the PTDPP-TT:PC ${ }_{71} \mathrm{BM}$ blend at short (ps-ns) and long (ns- $\mu \mathrm{s})$ delay times, respectively. The spectra on both timescales exhibit very similar features: (i) a broad and structured positive signal from 650 to $900 \mathrm{~nm}$ and (ii) a negative signal after the zero crossing at $900 \mathrm{~nm}$, which extends further into the NIR wavelength range beyond the spectral detection limit, which finally evolves into a PA peaking at $1150 \mathrm{~nm}$. In line with the aforementioned early time pump-probe spectra obtained on pristine polymer films, we assigned the positive signal to the polymer's GSB. The PA signal 
that extends into the NIR and is observed at early delay times, for instance at $0.5 \mathrm{ps}$ (red curve, figure 3a), resembles the shape of the singlet exciton-induced absorption spectrum, as measured on a pristine PTDPP-TT polymer film. However, we note that the charge-induced absorption spectra, which we independently obtained on a pristine polymer film after oxidation with iodine and also after oxidation with $\mathrm{FeCl}_{3}$ (see figure S5), are similar in shape and spectral position to the singlet exciton-induced absorption spectrum, which complicates a clear assignment. Interestingly, the broad PA signal, more specifically the PA at wavelengths longer than $1250 \mathrm{~nm}$, decays very rapidly and the shape of the spectrum changes, resulting in a new peak emerging at $1150 \mathrm{~nm}$. A further analysis of the TA data matrix by evolving factor analysis (EFA, see figure S6) and multivariate curve resolution (MCR) analysis excluding the first $20 \mathrm{ps,} \mathrm{in} \mathrm{which} \mathrm{singlet} \mathrm{excitons} \mathrm{are} \mathrm{expected} \mathrm{to} \mathrm{be} \mathrm{still} \mathrm{present} \mathrm{in} \mathrm{the} \mathrm{polymer:fullerene}$ blend, indicates that two species are sufficient to describe the TA data on the $20 \mathrm{ps}-\mathrm{ns}$ timescale. The two component spectra determined by MCR analysis are shown in figure S5, which also compares the component spectra with the separately measured triplet-induced absorption and charge-induced absorption spectrum obtained by control experiments. Component 1 is a broad and rather unstructured PA signal, which resembles very much the PA of charges seen in the control experiments. Component 2 can be clearly assigned to the triplet-induced absorption, as its spectral shape and position is almost the same as the tripletinduced absorption spectrum measured on the palladium-anthraporphyrin-doped polymer film. Figure $3 \mathrm{~d}$ shows the intensity dependence of the dynamics of the two components also obtained by MCR analysis. Interestingly, we observed a fluence-independent decay of component 1 (charges) which extends to $1 \mathrm{~ns}$, while component 2 (triplets) shows a moderate fluence dependent rise, that is, the onset of formation of component 2 shifts to earlier times with increasing fluence as shown in figure $3 \mathrm{~d}$ and also supported by EFA (see figure $\mathrm{S6}$ ). We note that, as described above, after $20 \mathrm{ps,} \mathrm{that} \mathrm{is} \mathrm{the} \mathrm{starting} \mathrm{point} \mathrm{for} \mathrm{our} \mathrm{MCR} \mathrm{analysis,} \mathrm{all}$ singlet excitons should have either recombined to the ground state due to their short lifetime 
or should have been quenched by the fullerene, so that component 1 can be assigned to charges. Furthermore, the intensity independent decay of component 1 may point to fast geminate recombination of bound charge pairs, while the intensity dependence of the rise of component 2 implies that triplet states are formed by non-geminate recombination as very recently also reported for other low-bandgap polymer:fullerene blends. ${ }^{[21,22]} \mathrm{We}$ note however, that the precise mechanism of the fast recombination process observed in these blends could not be entirely clarified by our TA experiments. In fact, many parameters such as intermolecular distance at the interface ${ }^{[28]}$, the presence of interfacial dipoles ${ }^{[29]}$ energy level bending at the interface or molecular weight of the polymer impact the interfacial charge separation mechanism and efficiency. ${ }^{[30]}$ We also note that a meaningful description of the dynamics of charges and triplets obtained by MCR analysis using a set of coupled rate equations based on a photophysical model was not possible, as too many parallel and sequential processes, such as geminate plus non-geminate recombination, triplet formation, decay and annihilation, simply over-parameterized any model.

Further TA experiments that we performed in the ns- $\mu$ s time range show almost exclusively the triplet-induced absorption (see figure 3b) indicating that the majority of the free charges have recombined and collapsed into the polymer's triplet state on this timescale. We also determined that the triplet-induced absorption in the PTDPP-TT:fullerene blend decays with a lifetime of $8 \mathrm{~ns}$, which is similar to the $15 \mathrm{~ns}$ lifetime (see figure S3) obtained on the porphyrin-doped polymer film further supporting our assignment of this peak to triplet states and indicating that their recombination channel is not fundamentally different in the two samples. 

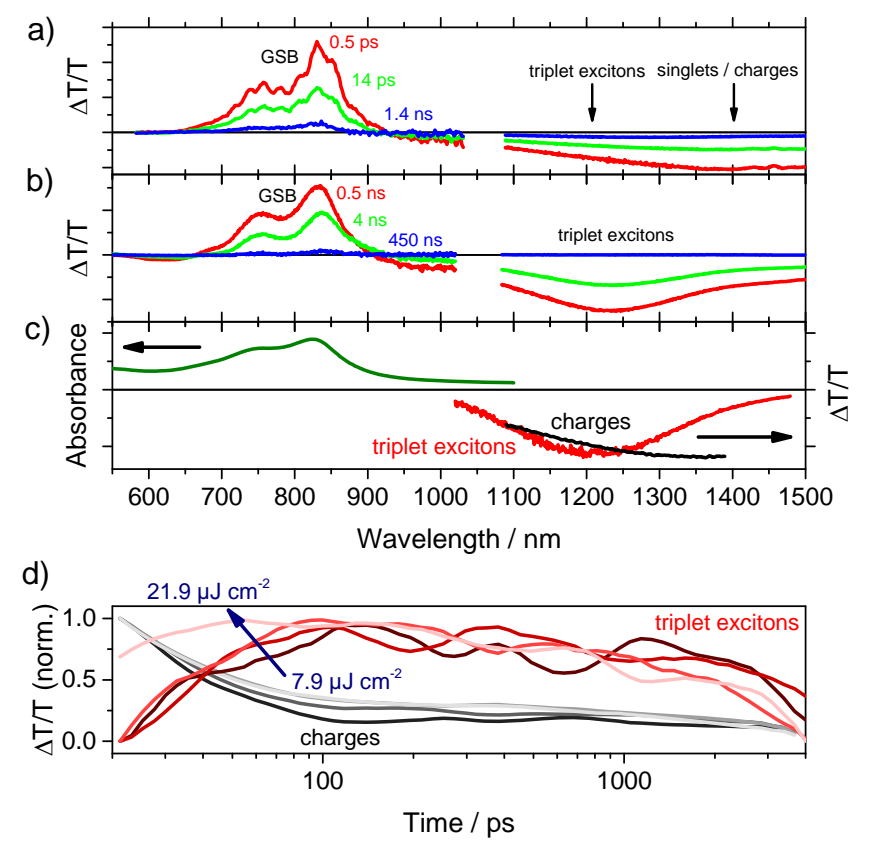

Figure 4. a) ps-ns Vis-NIR transient absorption spectra of a 1:2 (w/w) PFDPP-TT:PC ${ }_{71} B M$ blend at delay times of $0.5 \mathrm{ps}$ (red), $14 \mathrm{ps}$ (green) and $1.4 \mathrm{~ns}$ (blue). The pump fluence at $800 \mathrm{~nm}$ was $40.4 \mu \mathrm{J} \mathrm{cm}^{-2}$ for the visible and $12.3 \mu \mathrm{J} \mathrm{cm}^{-2}$ for the NIR measurements. b) ns- $\mu$ s Vis-NIR transient absorption spectra of a 1:2 (w/w) PFDPP-TT:PC ${ }_{71} \mathrm{BM}$ blend at delay times of $0.5 \mathrm{~ns}$ (red), $4 \mathrm{~ns}$ (green) and $450 \mathrm{~ns}$ (blue). The pump fluence at $532 \mathrm{~nm}$ was $70.4 \mu \mathrm{J} \mathrm{cm}^{-2}$ for the visible and $61.6 \mu \mathrm{J} \mathrm{cm}^{-2}$ for the NIR measurements. c) Absorption spectrum of a PFDPP-TT:PC ${ }_{71} \mathrm{BM}$ blend film (dark green), charge-induced absorption spectrum as obtained from MCR analysis (black), and triplet-induced absorption spectrum (red) obtained on a palladium-anthraporphyrin-doped polymer film. d) Fluence dependence of the dynamics of charges (black) and triplet excitons (red) obtained by MCR analysis using the separately determined triplet-induced absorption spectrum as input parameter (compare panel c, red curve). The corresponding charge spectrum is shown in panel c (black curve). The data matrix was analyzed after $20 \mathrm{ps}$ to exclude contributions from singlet excitons.

In case of the PFDPP-TT:PC ${ }_{71} \mathrm{BM}$ system we observed very similar phenomena as shown in

figure 4. Here, the EFA analysis also yielded two components on the ps-ns timescale. In line with the TA data obtained on PTDPP-TT:PC ${ }_{71} \mathrm{BM}$ the broad and unstructured photoinduced absorption evolves into a PA peaking at $1240 \mathrm{~nm}$ as shown in figure 4a, which resembles the triplet-induced absorption obtained on a porphyrin-doped polymer film (see figure 4c). The effect is even more pronounced for the ns- $\mu$ s TA data, which already after 500 ps showed exclusively the triplet-induced absorption (see figure $4 \mathrm{~b}$ ) likely due to the higher pump 
fluence used in these experiments. Interestingly, we observed the same lifetime of $11 \mathrm{~ns}$ (see figure S4) for triplets in the polymer:fullerene blend as we obtained in the porphyrin-doped PFDPP-TT polymer film, indicating that once triplets have been created in the photovoltaic blend their decay mechanism is similar to the porphyrin-doped polymer film. As in the case of PTDPP-TT we also observed a significant fraction of fast largely intensity-independent sub-ns recombination, as obtained by the MCR analysis shown in figure $4 \mathrm{~d}$. The assignment of the triplet formation mechanism to a geminate or non-geminate recombination process is less clear than in the case of PTDPP-TT, as we only observed an earlier onset of triplet formation for the highest fluence. In fact, here the MCR analysis is likely affected by the limited signalto-noise ratio, as indicated by the oscillations of the triplet exciton population in time. However, given the structural similarity of the two polymers, it appears reasonable that the photophysical processes are not fundamentally different.

The fast and almost quantitative triplet formation observed in both polymer:fullerene blends is rather astonishing and not compatible with the power conversion efficiencies of the respective photovoltaic devices, specifically the photocurrents and high fill factors. In fact, it appears that at the comparably high charge carrier concentrations obtained by pulsed laser excitation in our TA experiments triplet formation is enhanced likely due to the non-geminate, that is, intensity-dependent character of the triplet state formation mechanism. However, the only moderate short-circuit photocurrents, but rather high fill factors observed in devices may be explained by a considerable fraction of fast geminate recombination that can be deduced from the intensity-independent decay of component 1 in the MCR analysis of the TA data of both polymer:fullerene systems. 


\section{Conclusions}

In this study we investigated the exciton and charge carrier dynamics as well as the device performance of two DPP-based low band-gap polymers blended with $\mathrm{PC}_{71} \mathrm{BM}$ as acceptor in bulk heterojunction solar cell devices. The polymers differ in their chemical structure, namely the replacement of the two thiophenes adjacent to the DPP-unit in PTDPP-TT by furan in PFDPP-TT. The device performance of solar cells built with either of the polymers is at around $2.4 \%$, while small differences in $V_{O C}, J_{S C}$ and $F F$ were observed. We demonstrated that upon photon absorption singlet excitons are created in the pristine polymer films that are very short-lived and exhibit lifetimes of less than 20 ps. Nevertheless, triplet states are created as secondary photoproducts likely via fast singlet fission. In polymer:fullerene blends we observed that a significant fraction of exciton dissociation creates charges, which rapidly recombine on a sub-ns timescale and thereby may limit the experimentally obtained photocurrent with respect to the maximum photocurrent that could theoretically be obtained from the photoactive layer. The exact reason for the considerable fraction of this sub-ns recombination process requires further investigation in this series of materials. Finally, we demonstrate that under the measurement conditions applied in our TA experiments, that is high fluence and thus high charge carrier density, the majority of charges recombines and successively populates the polymer triplet state. However, further experiments are required to unravel whether the triplet state formation observed in our TA experiments does also play a role in a device under solar illumination conditions which is different from the conditions used in TA experiments, as the charge carrier concentration is lower and the applied bias aids the extraction of charges from the photoactive layer. 


\section{Supporting Information}

Supporting Information is available from the Wiley Online Library or from the author. Questions regarding the material synthesis should be addressed to K.-S. Lee, while questions regarding the photophysics should be addressed to F. Laquai.

Acknowledgements:

F.L. thanks the Max Planck Society for funding the Max Planck Research Group. J.R.O. thanks the IRTG 1404 for funding. H.A. thanks the DAAD for a RISE scholarship. D.W.G. acknowledges a Kekulé scholarship of the Fonds der Chemischen Industrie (FCI).

Received: Month XX, XXXX;

Revised: Month XX, XXXX;

Published online:

DOI: $10.1002 /$ marc.

Keywords: DPP polymers, organic photovoltaics, charge generation, transient absorption spectroscopy, triplet state formation 


\section{References}

[1] D. Chandran, K.-S. Lee, Macromol. Res. 2013, 21, 272.

[2] J. C. Bijleveld, V. S. Gevaerts, D. Di Nuzzo, M. Turbiez, S. G. Mathijssen, D. M. de Leeuw, M. M. Wienk, R. A. Janssen, Adv. Mater. 2010, 22, E242.

[3] Y. N. Li, P. Sonar, L. Murphy, W. Hong, Energy Environ. Sci. 2013, 6, 1684.

[4] Z. Yi, X. Sun, Y. Zhao, Y. Guo, X. Chen, J. Qin, G. Yu, Y. Liu, Chem. Mater. 2012, 24, 4350 .

[5] W. Li, W. S. Roelofs, M. M. Wienk, R. A. Janssen, J. Amer. Chem. Soc. 2012, 134, 13787.

[6] W. Li, K. Hendriks, A. Furlan, A. Zhang, M. M. Wienk, R. A. J. Janssen, Chem. Commun. 2015.

[7] D. Bartesaghi, M. Turbiez, L. J. A. Koster, Org. Electron. 2014, 15, 3191.

[8] D. H. Wang, A. K. K. Kyaw, J.-R. Pouliot, M. Leclerc, A. J. Heeger, Adv. Energy Mater. 2014, 4, n/a.

[9] L. Ye, S. Zhang, W. Ma, B. Fan, X. Guo, Y. Huang, H. Ade, J. Hou, Adv. Mater. 2012, $24,6335$.

[10] J. C. Bijleveld, A. P. Zoombelt, S. G. J. Mathijssen, M. M. Wienk, M. Turbiez, D. M. de Leeuw, R. A. J. Janssen, J. Am. Chem. Soc. 2009, 131, 16616.

[11] W. Li, K. H. Hendriks, A. Furlan, W. S. C. Roelofs, M. M. Wienk, R. A. J. Janssen, J. Am. Chem. Soc. 2013, 135, 18942.

[12] K. H. Hendriks, G. H. L. Heintges, V. S. Gevaerts, M. M. Wienk, R. A. J. Janssen, Angew. Chem. Int. Ed. 2013, 52, 8341.

[13] W. Li, A. Furlan, K. H. Hendriks, M. M. Wienk, R. A. J. Janssen, J. Am. Chem. Soc. 2013, 135, 5529.

[14] K. H. Hendriks, G. H. L. Heintges, M. M. Wienk, R. A. J. Janssen, J. Mater. Chem. A 2014, 2, 17899.

[15] A. P. Zoombelt, S. G. J. Mathijssen, M. G. R. Turbiez, M. M. Wienk, R. A. J. Janssen, J. Mater. Chem. 2010, 20, 2240.

[16] V. S. Gevaerts, A. Furlan, M. M. Wienk, M. Turbiez, R. A. Janssen, Adv. Mater. 2012, $24,2130$.

[17] S. Kouijzer, S. Esiner, C. H. Frijters, M. Turbiez, M. M. Wienk, R. A. J. Janssen, Adv. Energy Mater. 2012, 2, 945.

[18] W. Li, K. H. Hendriks, W. S. Roelofs, Y. Kim, M. M. Wienk, R. A. Janssen, Adv. Mater. 2013, 25, 3182.

[19] S. D. Dimitrov, A. A. Bakulin, C. B. Nielsen, B. C. Schroeder, J. Du, H. Bronstein, I. McCulloch, R. H. Friend, J. R. Durrant, J. Amer. Chem. Soc. 2012, 134, 18189.

[20] S. D. Dimitrov, C. B. Nielsen, S. Shoaee, P. Shakya Tuladhar, J. Du, I. McCulloch, J. R. Durrant, J. Phys. Chem. Lett. 2012, 3, 140.

[21] A. Rao, P. C. Chow, S. Gelinas, C. W. Schlenker, C. Z. Li, H. L. Yip, A. K. Jen, D. S. Ginger, R. H. Friend, Nature 2013, 500, 435.

[22] F. Etzold, I. A. Howard, N. Forler, A. Melnyk, D. Andrienko, M. R. Hansen, F. Laquai, Energy Environ. Sci. 2015, DOI: 10.1039/C4EE03630A.

[23] S. D. Dimitrov, S. Wheeler, D. Niedzialek, B. C. Schroeder, H. Utzat, J. M. Frost, J. Yao, A. Gillett, P. S. Tuladhar, I. McCulloch, J. Nelson, J. R. Durrant, Nat. Comm. 2015, 6, 6501.

[24] Y. W. Soon, H. Cho, J. Low, H. Bronstein, I. McCulloch, J. R. Durrant, Chem. Commun. 2013, 49, 1291.

[25] I. A. Howard, R. Mauer, M. Meister, F. Laquai, J. Amer. Chem. Soc. 2010, 132, 14866.

[26] F. Etzold, I. A. Howard, R. Mauer, M. Meister, T. D. Kim, K. S. Lee, N. S. Baek, F. Laquai, J. Amer. Chem. Soc. 2011, 133, 9469. 
[27] I. A. Howard, H. Mangold, F. Etzold, D. Gehrig, F. Laquai, "Transient absorption data analysis by soft-modeling", in Ultrafast Dynamics in Molecules, Nanostructures and Interfaces, World Scientific, 2013.

[28] T. W. Holcombe, J. E. Norton, J. Rivnay, C. H. Woo, L. Goris, C. Piliego, G. Griffini, A. Sellinger, J.-L. Brédas, A. Salleo, J. M. J. Fréchet, J. Amer. Chem. Soc. 2011, 133, 12106.

[29] C. Poelking, M. Tietze, C. Elschner, S. Olthof, D. Hertel, B. Baumeier, F. Würthner, K. Meerholz, K. Leo, D. Andrienko, Nat. Mater. 2015, 14, 434.

[30] R. C. Coffin, J. Peet, J. Rogers, G. C. Bazan, Nat. Chem. 2009, 1, 657. 
The exciton and charge carrier recombination processes in two DPP-polymers and their blends with fullerene are revealed by transient absorption spectroscopy. Both polymers exhibit very short exciton lifetimes. In blends exciton dissociation is followed by fast sub-ns charge recombination, in turn leading to a significant population of the polymer's triplet state, which are dominant on the ns- $\mu$ s timescale.

Julian Robert Ochsmann, Deepak Chandran, Dominik W. Gehrig, Husna Anwar, Pramod Kandoth Madathil, Kwang-Sup Lee,* and Frédéric Laquai*

Triplet state formation in photovoltaic blends of DPP-type copolymers and $\mathrm{PC}_{71} \mathrm{BM}$
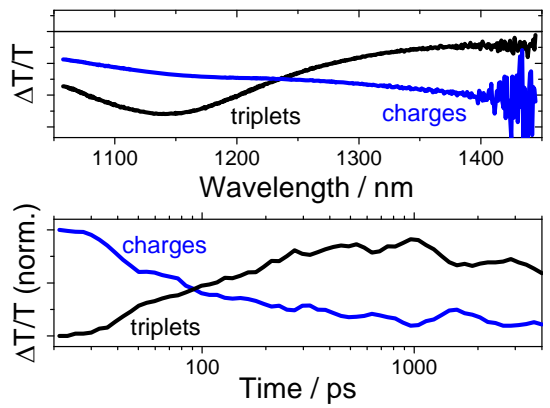